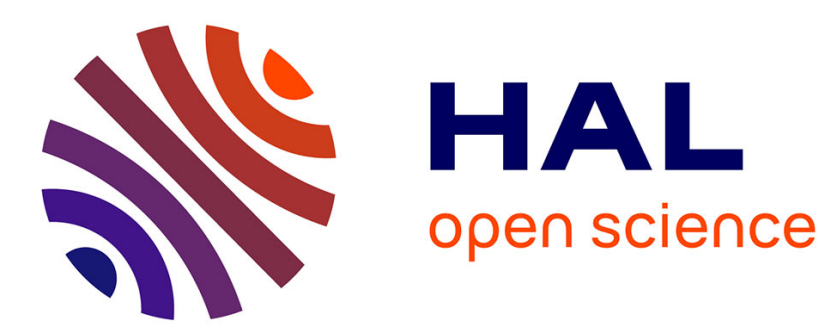

\title{
Grammaire comparée et langues romanes : la discussion méthodologique autour du Dictionnaire Étymologique Roman (DÉRom)
}

Eva Buchi

\section{- To cite this version:}

Eva Buchi. Grammaire comparée et langues romanes : la discussion méthodologique autour du Dictionnaire Étymologique Roman (DÉRom). Comptes-rendus des séances de l'Académie des inscriptions et belles-lettres, 2014, 2014 (1), pp.397-421. halshs-01180212

\section{HAL Id: halshs-01180212 \\ https://shs.hal.science/halshs-01180212}

Submitted on 24 Jul 2015

HAL is a multi-disciplinary open access archive for the deposit and dissemination of scientific research documents, whether they are published or not. The documents may come from teaching and research institutions in France or abroad, or from public or private research centers.
L'archive ouverte pluridisciplinaire HAL, est destinée au dépôt et à la diffusion de documents scientifiques de niveau recherche, publiés ou non, émanant des établissements d'enseignement et de recherche français ou étrangers, des laboratoires publics ou privés. 


\section{Comptes rendus de l'Académie des Inscriptions et Belles-Lettres 2014/1 : 397-421.}

GRAMMAIRE COMPARÉE ET LANGUES ROMANES : LA DISCUSSION MÉTHODOLOGIQUE

AUTOUR DU DICTIONNAIRE ÉTYMOLOGIQUE ROMAN (DÉROM)

\section{Présentation des enjeux du débat}

Monsieur le Secrétaire Perpétuel, Monsieur le Président, Mesdames et Messieurs les Académiciens, chers collègues, Mesdames, Messieurs, je voudrais remercier très vivement l'Académie des Inscriptions et Belles-Lettres de l'occasion qu'elle m’offre d'évoquer un débat méthodologique qui agite actuellement la communauté des linguistes romanistes, mais demeure assez peu connu en dehors de cette sous-discipline ${ }^{1}$.

L'étymologie romane, dont les origines remontent au milieu du $\mathrm{XIX}^{\mathrm{e}}$ siècle ${ }^{2}$, peut se féliciter d'un riche éventail d'ouvrages de référence, le plus éminent d'entre eux étant le Romanisches Etymologisches Wörterbuch $(\mathrm{REW})^{3}$ de Wilhelm Meyer-Lübke, dont la première édition parut en fascicules entre 1911 et 1920 et dont l'édition définitive, toujours dans le commerce, remonte à 1935.

Durant la seconde moitié du siècle passé, le bastion REW connut plusieurs vagues d'assaut, dont aucune ne fut pourtant couronnée de succès : ni le «nouveau REW » lancé dans les années cinquante par Harri Meier et Joseph Maria Piel (Piel 1961) sous l'égide de la DFG (Deutsche Forschungsgemeinschaft), ni le projet soumis à la DFG par Heinz Jürgen Wolf en 1984 (cf. Pfister 2013b, p. 132), ni le Petit Lexique étymologique panroman (cf. Chambon 1998, p. 1020) dont la mise en route fut décidée à l'issue de la table ronde « È oggi possibile o augurabile un nuovo REW ? » (Chambon et Sala 1998) du XXI ${ }^{\mathrm{e}}$ Congrès international de linguistique et de philologie romanes, qui se tint en 1995 à Palerme. Mais, quand Wolfgang Schweickard, professeur de philologie romane à l’Université de la Sarre (Sarrebruck), et moimême présentâmes en 2007, à l'occasion du $\mathrm{XXV}^{\mathrm{e}}$ Congrès international de linguistique et de philologie romanes d'Innsbruck, le projet d'un Dictionnaire Étymologique Roman (DÉRom ${ }^{4}$, cf. Buchi et Schweickard 2010), il existait depuis plusieurs générations déjà un consensus fort pour souhaiter la mise sur le chantier d’un nouveau dictionnaire étymologique panroman, de sorte que l'accueil de la communauté des romanistes fut très favorable.

\footnotetext{
${ }^{1}$ Mes remerciements les plus chaleureux s'adressent à Jean-Pierre Chambon (Paris), à Marco Maggiore (Nancy), à Robert Martin (Vice-Président de l'Académie des Inscriptions et Belles-Lettres, Paris), à Simone Pisano (Sassari) et à Fernando Sánchez Miret (Salamanque) pour leurs remarques stimulantes sur une première version de ce texte.

${ }^{2}$ On peut faire commencer l'époque scientifique de l'étymologie romane avec le Etymologisches Wörterbuch der romanischen Sprachen de Friedrich Diez (1953).

${ }^{3}$ REW = W. Meyer-Lübke, Romanisches Etymologisches Wörterbuch, Heidelberg, 1930-1935 ${ }^{3}\left(1911-1920^{1}\right)$.

${ }^{4}$ DÉRom = É. Buchi et W. Schweickard (dir.), Dictionnaire Étymologique Roman (DÉRom). Nancy, 2008-, http://www.atilf.fr/DERom. - Le DÉRom bénéficie actuellement (2012-2014) pour la seconde fois d'une subvention de la part de l'ANR (ANR-11-FRAL-0008) et de la DFG.
} 
Tout en se définissant comme une œuvre de fidélité vis-à-vis de Meyer-Lübke - et d'ailleurs vis-à-vis de l'ensemble de la tradition extrêmement riche des études de linguistique et de philologie romanes -, le DÉRom se distingue de son illustre prédécesseur sur un point de méthode central : là où les étymons du REW sont constitués d'unités lexicales latines (ou, quand les attestations textuelles font défaut, "pseudo-latines $\left.»^{5}\right)^{6}$, ceux du DÉRom sont reconstruits selon la méthode de la grammaire comparée, dont l'expression classique se trouve dans La méthode comparative en linguistique historique d'Antoine Meillet (1925) ${ }^{7}$. Traditionnellement, cette méthode pour ainsi dire hégémonique en étymologie indoeuropéenne ou afro-asiatique, mais aussi germanique ou slave, était réputée de peu d’intérêt pour l'étymologie romane en raison du témoignage massif du latin écrit. Si le DÉRom rompt avec cette approche séculaire et pose la reconstruction comparative au centre de sa démarche, c'est en application du programme dressé par Jean-Pierre Chambon dans deux publications appelées à faire date (Chambon 2007 ; 2010), et dont le message central se dégage des lignes suivantes :

«[...] les mots du latin écrit de l’Antiquité ne sauraient être placés à l'origine des mots héréditaires du français ou des autres langues (gallo)romanes [...]. Le seul moyen de faire venir à l'existence l'étymon (oral) d'un mot héréditaire est de le reconstruire sur la base de la comparaison entre formes orales affines [...]. On dira donc que l'établissement des étymons des mots héréditaires est le segment de la recherche étymologique où celle-ci coïncide avec la grammaire comparée-reconstruction. » (Chambon 2010, p. 64.)

Cette option méthodologique du DÉRom, dont Wolfgang Schweickard et moi-même avions très largement sous-évalué le potentiel explosif, a été de nature à projeter le projet au centre d'une discussion paradigmatique dont le point de départ peut être daté du 5 septembre 2007, date à laquelle le projet fut présenté lors du congrès de linguistique romane d’Innsbruck, qui culmina avec la table ronde consacrée au DÉRom lors du congrès de linguistique romane de Valence de 2010 (Pfister 2013a) et qui, malgré certains signes d’apaisement (Greub 2014, p. 270), ne semble pas près de s’essouffler.

Comme toute discussion, celle-ci a comporté sa part de malentendus inévitables (Buchi et Schweickard 2011a; cf. aussi 2011b). Il n'en reste pas moins que le recours à la reconstruction comparative pour l'établissement des étymons du lexique héréditaire roman est loin de faire l'unanimité, comme en témoignent les prises de position fortement critiques vis-

\footnotetext{
${ }^{5}$ Pour ces étymons en « "fiddled with” classical Latin », cf. Buchi 2010, p. 2.

${ }^{6}$ Précisons que cela n’est vrai que pour le lexique héréditaire, la seule classe étymologique traitée par le DÉRom ; le REW contient par ailleurs des étymons réputés arabes (mugāwir), basques (gazmuña), franciques (stal), gaulois (bascauda), grecs (tartarūchos), néerlandais (prik), etc.

${ }^{7}$ Pour la proto-histoire de la méthode comparative, $c f$. Baxter 2002.
} 
à-vis de cette option méthodologique de la part de quatre très grands noms de la linguistique romane : Germà Colón, un des maîtres de la lexicologie romane et catalane, Johannes Kramer, auteur notamment du grand dictionnaire étymologique du ladin dolomitique ${ }^{8}$ (Kramer 2011a ; 2011b), Frankwalt Möhren, ancien directeur du Dictionnaire Étymologique de l'Ancien Français (Möhren 2012), et surtout le regretté Alberto Vàrvaro, auteur notamment du dictionnaire étymologique du sicilien $(\mathrm{VSES})^{9}$ (Vàrvaro 2011a ; 2011b), qui exprima son opposition de principe de façon particulièrement virulente ${ }^{10}$. Ces auteurs contestent unanimement la légitimité de la méthode comparative en étymologie romane, ainsi Alberto Vàrvaro :

- « [...] una cosa è ricostruire su base comparativa, mettiamo, il germanico e tutt’altra cosa è applicare lo stesso metodo alle lingue romanze ed al latino. Nel primo caso non abbiamo nessuna documentazione della lingua che intendiamo studiare [...]. Applicare al caso del latino e delle lingue romanze la metodologia che si impone (per ragioni di fatto, non per nostra scelta) nel caso dell'indoeuropeo e delle lingue indoeuropee preistoriche sarebbe come studiare la storia della Francia napoleonica con i metodi normali in preistoria. » (Vàrvaro 2011a, p. 299-300.)

Johannes Kramer, quant à lui, déplore le caractère « exsangue » des étymons reconstruits :

- «[...] damit hat man aber das Etymon im eigentlichen Sinne, also das Element, das im Lateinischen in irgendeiner seiner Erscheinungsformen real existierte, ein reales semantisches Spektrum aufwies und eine reale Einbindung in die sprachlich-reale Umwelt aufwies, in die zweite Reihe verwiesen und arbeitet [man] innerromanistisch nur noch mit blutleeren Rekonstruktions-Etyma. » (Kramer 2011a, p. 779¹.)

Pour Frankwalt Möhren, les témoignages écrits devraient occuper le devant de la scène :

- «Cette phrase-clé de la présentation du DÉRom (site de l’ATILF) contient un terme-clé qui est ancêtre commun. Il est vrai que les germanistes par exemple cherchent des ancêtres communs des langues transmises par des textes par la méthode comparative et donc reconstructive pour isoler des langues ancêtres qu'ils tâchent de localiser et de dater [...]. Les reconstructions [...] doivent coïncider avec les faits attestés [...], sinon elles ne seraient pas considérées comme scientifiques. [...] C’est indubitablement un fait que les langues

\footnotetext{
${ }^{8}$ EWD = J. Kramer (dir.), Etymologisches Wörterbuch des Dolomitenladinischen, 8 vol., Hambourg, 19881998.

${ }^{9}$ VSES = A. Vàrvaro, Vocabolario Storico-Etimologico del Siciliano, 2 vol., Strasbourg, 2014.

${ }^{10} C f$. aussi, malgré une prise de position globalement très favorable au DÉRom, Pfister 2013b, p. 134 : «la seule goutte d'amertume pour moi, c'est la base protoromane. Si nous disposons d'une base lexicologique excellente, le Thesaurus Linguae Latinae et de bons dictionnaires médiolatins, je me demande pourquoi cette base fictive avec des astérisques [...]».

${ }^{11}$ Cf. aussi Kramer 2011b, p. 199 : «eine im Grunde nutzlose Ebene ist die des Protoromanischen, das mit Mitteln der Rekonstruktion aus der Rückprojizierung der Gegebenheiten der romanischen Sprachen in die Vergangenheit gewonnen wird ».
} 
romanes descendent d'une langue commune, mais il nous intéresse vraiment de savoir si la reconstruction faite selon la mécanique phonétique de Fox, combinée avec les vues de de Dardel, aura les qualités d'une langue, par exemple d'avoir une ou des synchronies, posséder des variétés, etc. etc. » (Möhren 2012, p. 9 ; 10 ; 12.)

Et surtout, à l'instar de Germà Colón, ces auteurs appellent de leurs vœux un retour à des lemmes « latins » :

- « [...] la presentació en alfabet fonètic internacional em sembla desencertada en una obra que duu al títol l’adjectiu roman. Ultra això allunyaria el públic culte no romanista. Almenys hi hauria d’haver l’ètim llatí com a lema » (Colón 2013, p. 150.)

Quant au latiniste Xaverio Ballester, qui s’exprime de façon similaire, il critique notamment le caractère « abstrait, éthéré et non documenté » de la protolangue reconstruite :

- « Si, por ejemplo, para el subvencionadísimo proyecto internacional de un Dictionnaire Étymologique Roman "l’objectif déclaré [...] consiste à reconstruire le lexique de l'ancêtre commun des parlers romans : le protoroman” (Buchi \& Schweickard $2009: 100$ [...]), en vez de protoroman $\dot{¿}$ no habría que decir latin commun para señalar precisamente que se estudia sólo la antigua base común de las lenguas románicas ? [...] Parece, pues, que estos protoromanistes aspiran a crear una prelengua románica que no se corresponda con el latín, entre otras cosas, por su concepto tan restrictivo del latín que actuó de base en las lenguas románicas y que en la práctica es para ellos algo más abstracto, etéreo e indocumentado que aquel tradicional latín vulgar. » (Ballester 2014, p. 83.)

La ligne de partage entre adeptes et détracteurs de la méthode déromienne s’opère grosso modo (il y a des exceptions !) selon les trois paramètres suivants : premièrement, les plus jeunes ont tendance à adhérer massivement au nouveau paradigme, tandis que l'opposition la plus forte provient de seniores ; deuxièmement, ceux qui se perçoivent prioritairement comme des linguistes ont nettement plus tendance à se reconnaître dans le nouveau paradigme que ceux qui se définissent prioritairement comme des philologues; enfin, les linguistes romanistes pour qui la linguistique générale représente la « discipline-toit » de leur activité scientifique adhèrent beaucoup plus volontiers au nouveau paradigme que ceux pour qui la romanistique (dans le sens « étude des langues et des littératures romanes ») joue ce rôle ${ }^{12}$.

\section{Illustration des deux approches par des exemples concrets}

Après avoir esquissé les deux positions théoriques qui s’affrontent dans la discussion paradigmatique actuelle, je me propose d’illustrer le débat par des exemples concrets tirés du

\footnotetext{
${ }^{12}$ Parmi les personnalités qui adhèrent au nouveau paradigme, nombreuses sont d'ailleurs celles qui ont décidé de rejoindre les rangs du DÉRom, dont l'équipe réunit aujourd’hui plus de cinquante chercheurs implantés dans treize pays européens (Allemagne, Belgique, Croatie, Espagne, France, Italie, Portugal, République de Macédoine, République tchèque, Roumanie, Russie, Slovénie, Suisse) et trois pays d’outre-mer (Brésil, États-Unis, Japon).
} 
REW et du DÉRom. Concernant ce dernier, la base documentaire sera constituée des environ trois cents articles rédigés, dont 114, qui ont parcouru l'ensemble des étapes de révision, sont accessibles sur internet. J'évoquerai tour à tour les propriétés phonétiques et phonologiques des étymons, leurs propriétés sémantiques, enfin leurs propriétés morphosyntaxiques.

\subsection{PROPRIÉTÉS PHONÉTIQUES ET PHONOLOGIQUES DES ÉTYMONS}

Le traitement étymologique de fr. vendre v.ditr. « céder (à qn) la propriété (de qch.) pour un certain prix » et de ses cognats nous servira d'exemple pour la mise en évidence des différences qui frappent les étymons mis en avant par les deux méthodes sur le plan phonique.

On trouvera en annexe, sous 5.1., les articles vēnděre du REW de Meyer-Lübke (REW 3 1935) et */'ßend-e-/ du DÉRom (Groß 2014 in DÉRom). Le moins que l’on puisse dire, c'est que la reconsidération, à quelque quatre-vingts ans d'intervalle, de l'étymologie de fr. vendre et de ses cognats sarde, roumain, italien, espagnol ou encore portugais n'aboutit pas à un résultat révolutionnaire : quand on considère les deux étymons mis en avant par le REW et le DÉRom, vēnděre et */'ßend-e-/, on constate (1) que leurs signifiants sont très proches, en tout cas quand on s'en tient à l'étymon direct que le DÉRom cite au début des matériaux, qui est, comme dans le REW, un mot-forme (en l'occurrence un infinitif), tandis que le signifiant de l'étymon qui apparaît dans le lemme présente la forme plus abstraite d’un lexème ; (2) que leurs signifiés sont interchangeables, même si à la simple glose du REW s'oppose, dans le DÉRom, une définition componentielle ; (3) que la catégorie grammaticale que le DÉRom attribue à l'étymon est implicitement présente dans le REW aussi. Tout au plus pourrait-on accorder à l'article du DÉRom le mérite de contenir une étymologisation - en l'occurrence, réduite à sa plus simple expression - explicite («tous les parlers romans sans exception présentent des cognats conduisant à reconstruire protorom. */'ßend-e-/ [...]»), condition nécessaire à sa validation ou son invalidation. Ce ne sont certainement pas des articles de ce type qui illustrent le mieux la plus-value en termes de connaissances factuelles de l’application de la reconstruction comparative à l'étymologie romane.

Toutefois, du point de vue des propriétés phonétiques et phonologiques de l'étymon, le lemme du REW, sous sa forme empruntée aux textes latins (et assorti de diacritiques de quantité vocalique), pose problème. En effet, pour ne mentionner que cet exemple, quelle est la valeur phonétique et phonologique du graphème $<\mathrm{v}>$ ? Si, comme les prises de position de Colón, Kramer, Möhren et Vàrvaro citées ci-dessus tendent à l’indiquer, le lemme relève du latin, la réponse réside du côté des études latines. En latin classique, le graphème $<\mathrm{v}>$ note, à l'initiale et devant consonne, le phone $[\mathrm{w}]$ et le phonème /u/ (Leumann 1963, p. $110 \S 99$; 
Maniet 1975, p. 24 § 5 ; 28 § 8 ; Väänänen 1981, p. 49 § 87 ; 50 § 89 ; Moralejo 1989 ; Serbat 1994, p. 33-34 ${ }^{13}$; Ballester 1996 ; Touratier 2005, p. 62-64).

Pour ce qui est de l'étymon du DÉRom, il contient une fricative bilabiale */ $\beta /$, que l'on reconstruit, en vertu du critère des traits phonétiques partagés, comme ancêtre commun de l'occlusive bilabiale des cognats sarde, gascon et espagnol (sard. /'bender/ ['bender(c)], esp. /ben'der/ [ben'der]) et de la fricative labiodentale /v/ des autres cognats romans (ainsi fr. ['vãds]) : le protophonème combine le trait 'fricatif' (mode d'articulation) d'un groupe de parlers et le trait 'bilabial' (lieu d'articulation) de l'autre. Les deux étymons se distinguent donc sur ce point au moins, et on peut en conclure que la méthode comparative n'est pas complètement inutile, car elle apporte un éclairage nouveau sur la phonie.

D’aucuns rétorqueront que raisonner ainsi, c'est faire peu de cas des connaissances implicites que les romanistes sont parfaitement capables de mobiliser à la lecture d'un dictionnaire étymologique, et qu'en l'occurrence, le lemme vēnděre est mentalement translittéré en ['ßendere], tant tout romaniste digne de ce nom a à l'esprit la destinée de [w] latin :

«Puis à partir du $1^{\mathrm{er}}$ siècle de notre ère, [...]. Dès cette époque $b$ et $w$ se confondent [...]. C'est que l'un et l'autre étaient passés à la constrictive bilabiale $\beta$ » (Väänänen 1981, p. $50 \S$ 89 ; cf. Weiss 2011, p. 512)

Il faut répondre : (1) qu'un dictionnaire étymologique des langues romanes ne s’adresse pas exclusivement aux romanistes; (2) que le latin ne fait plus nécessairement - et je le déplore ! - partie du bagage intellectuel de tous les étudiants en linguistique romane (pour peu que cette discipline soit encore enseignée et ne soit pas diluée dans des études de linguistique idioromanes) ; (3) que les résultats de recherches scientifiques se doivent d'être énoncés le plus clairement possible, sans obliger le lecteur à une gymnastique mentale dont le seul but serait de tester son appartenance à un cercle d’initiés.

\subsection{PROPRIÉTÉS SÉMANTIQUES DES ÉTYMONS}

Il est plus facile de mettre le doigt sur les différences qui frappent les étymons générés par les deux méthodes en concurrence quand on fait porter son attention sur les aspects sémantiques. En particulier, ce qui est en cause, c’est la polysémie des étymons. S’il est vrai qu'il existe des cas de non-concordance où l'ancienne méthode met en avant un sens que la nouvelle méthode interdit de poser (cf. Buchi 2013, p. 5-6), l'inverse est beaucoup plus fréquent : d'une manière générale, la reconstruction comparative incite à penser que le lexique de l'ancêtre des parlers romans était beaucoup plus polysémique que ce que l’on croyait.

\footnotetext{
${ }^{13}$ Serbat 1994, p. 33-34 accorde à [w] un statut de phonème, mais comme il le note entre crochets carrés (!), le doute est permis.
} 
Une illustration s'en trouve en annexe, sous 5.2., où ont été juxtaposés les articles tītio, -ōne du REW $\left(1935^{3}\right.$ ) et */ti'tion-e/ du DÉRom (Jactel et Buchi 2012/2013 in DÉRom). Pour le REW, l'étymon d'it. tizzone et de ses cognats est monosémique : il définit «Feuerbrand [tison]», alors que le DÉRom pose un étymon bisémique : «tison ; charbon (maladie des céréales) ».

En effet, le sens « maladie des céréales d’origine cryptogamique qui les convertit en poussière noirâtre, charbon » se reconstruit de cognats sarde, dacoroumain, aroumain, calabrais, frioulan, espagnol et asturien. Comme l'évolution sémantique qui mène de «tison » à «maladie des céréales » n’est pas triviale, il est extrêmement peu probable qu’elle soit intervenue de manière indépendante dans ces sept idiomes (ou bien, si l'on faisait l'hypothèse d'emprunts de l'aroumain au dacoroumain et de l'asturien à l'espagnol, dans cinq idiomes différents). On est donc en droit de reconstruire ce second sémème pour l'étymon qui constitue l'ancêtre commun de l'ensemble des parlers romans, et on expliquera l'absence de ce sens dans un certain nombre d'idiomes romans par des pertes intervenues à date prélittéraire.

Sur un plan plus général, on rappellera que la polysémie lexicale est une caractéristique universelle des langues naturelles : poser un étymon bisémique va donc dans le sens d'un système linguistique réel. Or, le sémème secondaire « charbon (maladie des céréales) » n’est pas attesté en latin écrit de l'Antiquité (ø $\mathrm{OLD}^{14}$ ), ce qui en explique l'absence dans le REW : les sens non transmis par les textes «constitue[nt] l'angle mort définitoire de la méthode traditionnelle » (Buchi 2012, p. 113).

\subsection{PROPRIÉTÉS MORPHOSYNTAXIQUES DES ÉTYMONS}

C’est la valence verbale, et plus précisément celle de l'étymon de fr. croître et de ses cognats, qui nous fournira l'exemplification des différences entre les résultats des deux méthodes en termes de propriétés morphosyntaxiques (cf. annexe 5.3.). À son habitude, le REW ne précise pas la catégorie grammaticale de son étymon, mais on déduit que crēscěre est un verbe intransitif de la glose dont il est assorti : «wachsen [croître] » (REW 1935³). Pour ce qui est du DÉRom, son étymon se caractérise par une valence double : */'kresk-e-/ v.intr. « grandir progressivement jusqu'au terme du développement normal, croître », tr. « rendre plus grand, accroître » (Maggiore 2011-2014 in DÉRom), l'emploi transitif étant reconstruit sur la base de cognats sarde, roumain, istriote, italien, frioulan, français, francoprovençal, occitan, gascon, catalan, espagnol, asturien et portugais. L'absence de cette valence dans le REW est,

\footnotetext{
${ }^{14}$ OLD = P. G. W. Glare (éd.), Oxford Latin Dictionary, Oxford, 1968-1982.
} 
là encore, consistante avec la méthode employée : le latin écrit ne connaissant pas la valence transitive pour crescere (ø TLL ${ }^{15}$ ), l’étymon n’est pas réputé la présenter non plus.

\section{Conclusion}

$\mathrm{Au}$ terme de ce rapide parcours, je suis confiante que même ceux qui ne partagent pas l'enthousiasme de notre équipe pour les potentialités qui s'ouvrent à l'étymologie romane grâce au recours à la reconstruction comparative reconnaîtront que les gains obtenus dans le cadre du DÉRom sont bien réels. Comment expliquer alors cette levée de boucliers de l'establishment de la linguistique romane, et cela avant même que le premier volume du dictionnaire ne soit paru ? On peut en voir, avec Jean-Pierre Chambon, les raisons profondes dans le rendez-vous manqué, structurel et structurant, entre grammaire comparée et linguistique romane. En effet :

«[...] bien avant qu'il n'existe des linguistes et des romanistes, les liens des langues romanes entre elles et avec le latin constituaient un fait de conscience épi- et métalinguistique déjà présent dans les représentations langagières et culturelles des classes cultivées romanophones [...]. La parenté étant pour elle une évidence culturelle héritée, la linguistique romane a jugé économique de ne pas mobiliser le lourd appareil démonstratif de la grammaire comparée. [...] Certes, la dimension de la comparaison est éminemment présente dans le 'paradigme romaniste', mais cette comparaison-là n'est pas la grammaire comparéereconstruction. Celle-ci reste au contraire [...] la grande exclue et, pour ainsi dire, le refoulé de la discipline. Disons que la comparaison des romanistes relève d'un état d'esprit, non d'une méthode et de techniques. C'est le refoulement - défense automatique par laquelle on rejette une idée pénible et/ou dangereuse en s'en dissociant - qui explique la vigueur des réactions du milieu romaniste dès qu'il est question de grammaire comparée-reconstruction romane. » (Chambon à paraître, p. 3-4.)

En tout état de cause, on ne peut que se féliciter de la coexistence des deux paradigmes que connaît désormais l'étymologie romane, le paradigme latinisant et le paradigme reconstructionniste. Succédant à une période de consensus un peu mou autour de l'idée d'une étymologie romane radicalement différente des étymologies des autres langues du monde, qui pourrait se contenter d'emprunter ses résultats de recherche aux dictionnaires latins, la période que nous vivons a quelque chose de vivifiant : un monopole a été brisé, et d'un coup, grâce à un changement de point de vue radical, tout semble possible. Toutefois, il ne saurait être question de vouloir remplacer l'ancien monopole par un nouveau : la discussion entre tenants de l'ancienne et de la nouvelle méthode doit se poursuivre. Pour cette raison, Wolfgang Schweickard et moi-même avons invité Johannes Kramer, qui, malgré son opposition de

\footnotetext{
${ }^{15}$ TLL = Thesaurus Linguae Latinae, Leipzig/Stuttgart/Berlin/New York, 1900-.
} 
principe à sa méthode, est un membre dévoué du DÉRom, à contribuer un chapitre intitulé «Contrepoint: ce que j’aurais fait différemment dans le DÉRom » au premier volume du dictionnaire (Kramer 2014). Le prochain épisode, dont on devine d'ores et déjà l’intérêt, sera constitué par l'accueil que la communauté scientifique - celle des romanistes, mais aussi plus généralement celle des linguistes - réservera à ce premier volume du dictionnaire : nul doute que l'éventail entre adhésion complète et opposition franche sera large. En attendant, le débat entre détracteurs et défenseurs du DÉRom aura joué un rôle très positif, tant au sein même du projet, il a permis d'atteindre une position plus équilibrée ${ }^{16}$.

\section{Bibliographie}

X. Ballester, «Fonemática de /u/ en latín. El argumento de los pares mínimos », Faventia 18/2 (1996), p. 137-140.

X. Ballester, «Protoroman, la lengua de nunca jamás », dans Linguistique romane et linguistique indo-européenne. Mélanges offerts à Witold Manczak à l'occasion de son 90 anniversaire, Cracovie, 2014, p. 77-86.

W. H. Baxter, «Where does the 'comparative method' come from ? », dans The Linguist's Linguist. A Collection of Papers in Honour of Alexis Manaster Ramer, F. Cavoto (éd.), Munich, 2002, vol. 1, p. 33-52.

M. Benarroch et E. Baiwir, «Reconstruction flexionnelle», dans Le Dictionnaire Étymologique Roman (DÉRom). Genèse, méthodes et résultats, É. Buchi et W. Schweickard (éd.), Berlin, 2014, p. 129-165.

É. Buchi, «Where Caesar’s Latin does not belong : a comparative grammar based approach to Romance etymology », dans Selected Proceedings of the Fifth International Conference on Historical Lexicography and Lexicology held at St Anne's College, Oxford, 16-18 June 2010, Ch. Brewer (éd.), Oxford, 2010, http://ora.ox.ac.uk/objects/uuid\%3A237856e6a327-448b-898c-cb1860766e59.

É. Buchi, « Des bienfaits de l'application de la méthode comparative à la matière romane : l'exemple de la reconstruction sémantique », dans Methods of Etymological Practice, B. Vykypěl et V. Boček (éd.), Prague, 2012, p. 105-117.

É. Buchi, «Les langues romanes sont-elles des langues comme les autres ? Ce qu'en pense le DÉRom », communication inédite présentée au XXVII Congrès international de linguistique et de philologie romanes (Nancy, 15-20 juillet 2013). Section 1 : Linguistique générale/linguistique romane, 2013.

É. Buchi et W. Schweickard, « À la recherche du protoroman : objectifs et méthodes du futur Dictionnaire Étymologique Roman (DÉRom) », dans Actes du XXV $V^{e}$ Congrès International

${ }^{16}$ Cf. Maggiore et Buchi 2014 pour une tentative de pondération entre les deux principaux moyens de connaissance du latin. 
de Linguistique et de Philologie Romanes (Innsbruck 2007), M. Iliescu, H. SillerRunggaldier et P. Danler (éd.), Berlin/New York, 2010, vol. 6, p. 61-68.

É. Buchi et W. Schweickard, «Sept malentendus dans la perception du DÉRom par Alberto Vàrvaro », Revue de linguistique romane 75 (2011), p. 305-312 (= 2011a).

É. Buchi et W. Schweickard, «Ce qui oppose vraiment deux conceptions de l'étymologie romane. Réponse à Alberto Vàrvaro et contribution à un débat méthodologique en cours », Revue de linguistique romane 75 (2011), p. 628-635 (= 2011b).

J.-P. Chambon, « Conclusions partielles/conclusion générale», dans Chambon et Sala 1998, p. 1017-1020.

J.-P. Chambon, «Remarques sur la grammaire comparée-reconstruction en linguistique romane (situation, perspectives) », Mémoires de la Société de linguistique de Paris 15, 2007, p. 57-72.

J.-P. Chambon, « Pratique étymologique en domaine (gallo)roman et grammaire comparéereconstruction. À propos du traitement des mots héréditaires dans le TLF et le FEW », dans Typologie et comparatisme. Hommages offerts à Alain Lemaréchal, I. Choi-Jonin, M. Duval et O. Soutet (éd.), Louvain/Paris/Walpole, 2010, p. 61-75.

J.-P. Chambon, «Réflexions sur la reconstruction comparative en étymologie romane : entre Meillet et Herman », dans Étymologie romane. Objets, méthodes et perspectives, M.-D. Gleßgen et W. Schweickard (éd.), Strasbourg, à paraître.

J.-P. Chambon et M. Sala (dir.). « Tavola rotonda. È oggi possibile o augurabile un nuovo REW ? », dans Atti del XXI Congresso Internazionale di Linguistica e Filologia Romanza (Centro di studi filologici e linguistici siciliani, Università di Palermo 18-24 settembre 1995), G. Ruffino (éd.), Tübingen, 1998, vol. 3, p. 983-1023.

G. Colón, «[Intervention à la table ronde "100 anys d'etimologia romànica : el REW de Meyer-Lübke : 1911-2010”] », dans Pfister 2013a, p. 150.

Y. Greub, «Débat méthodologique », dans Le Dictionnaire Étymologique Roman (DÉRom). Genèse, méthodes et résultats, É. Buchi et W. Schweickard (éd.), Berlin, 2014, p. 269-288.

J. Kramer, « Tolle grabattum tuum und betreibe kulturwissenschaftliche Etymologie ! », dans Lexikon, Varietät, Philologie. Romanistische Studien Günter Holtus zum 65. Geburtstag, A. Overbeck, W. Schweickard et H. Völker (éd.), Berlin/Boston, 2011a, p. 769-781.

J. Kramer, "Latein, Proto-Romanisch und das DÉRom », Romanistik in Geschichte und Gegenwart 17, 2011b, p. 195-206.

J. Kramer, «Contrepoint : ce que j’aurais fait différemment dans le DÉRom », dans Le Dictionnaire Étymologique Roman (DÉRom). Genèse, méthodes et résultats, É. Buchi et W. Schweickard (éd.), Berlin, 2014, p. 289-297. 
M. Leumann, Lateinische Grammatik, vol. 1 : Lateinische Laut- und Formenlehre, Munich, 1963 [1926-1928].

M. Maggiore et É. Buchi, « Le statut du latin écrit de l'Antiquité en étymologie héréditaire française et romane ", dans Actes du Congrès Mondial de Linguistique Française 2014 (Berlin, 19-23 juillet 2014), F. Neveu, P. Blumenthal, L. Hriba, A. Gerstenberg, J. Meinschaefer et S. Prévost (éd.), Paris, http://dx.doi.org/10.1051/shsconf/20140801161, 313-325.

A. Maniet, La phonétique historique du latin dans le cadre des langues indo-européennes, Paris, $1975^{5}\left(1950^{1}\right)$.

A. Meillet, La méthode comparative en linguistique historique, Oslo et al., 1925.

Fr. Möhren, «Édition, lexicologie et l'esprit scientifique », dans Present and future research in Anglo-Norman. Proceedings of the Aberystwyth Colloquium, 21-22 July 2011, D. Trotter (éd.), Aberystwyth, 2012, p. 1-13.

J. L. Moralejo, «Fonética y fonología de v en latín clásico », dans Actas del VII Congreso español de estudios clásicos (Madrid, 20-24 de abril de 1987), Madrid, 1989, p. 511-516.

M. Pfister (dir.), «Taula redona : 100 anys d'etimologia romànica : el REW de MeyerLübke : 1911-2010 », dans Actas del XXVI Congreso Internacional de Lingüística y de Filología Románicas (Valencia 2010), E. Casanova Herrero et C. Calvo Rigual (éd.), Berlin/New York, 2013a, vol. 1, p. 131-158 (= 2013a).

M. Pfister, « Presentació », dans Pfister 2013a, p. 131-134 (= 2013b).

J. M. Piel, «De l'ancien REW au nouveau REW », dans Lexicologie et lexicographie françaises et romanes. Orientations et exigences actuelles (Strasbourg, 12-16 novembre 1957), Paris, 1961, p. 221-239.

G. Serbat, Les structures du latin. Avec un choix de textes traduits et annotés de Plaute aux Serments de Strasbourg, Paris, $1994^{4}\left(1975^{1}\right)$.

Chr. Touratier, "Système des consonnes ", dans Essais de phonologie latine. Actes de l'atelier d'Aix-en-Provence 12-13 avril 2002, Chr. Touratier (éd.), Aix-en-Provence, 2005, p. $61-134$.

V. Väänänen, Introduction au latin vulgaire, Paris, $1981^{3}\left(1963^{1}\right)$.

A. Vàrvaro, «Il DÉRom : un nuovo REW? », Revue de linguistique romane 75, 2011, p. 297304 (= 2011a).

A. Vàrvaro, «La 'rupture épistémologique’ del DÉRom. Ancora sul metodo dell'etimologia romanza », Revue de linguistique romane 75, 2011, p. 623-627 (= 2011b).

M. Weiss, Outline of the Historical and Comparative Grammar of Latin, Ann Arbor/New York, $2011^{2}\left(2009^{1}\right)$. 


\section{Annexes}

\subsubsection{Article vēnděre du $\mathrm{REW}_{3}$}

9190. vēnděre «verkaufen»

Rum. vinde, vegl. vandro, it. vendere, log. bendere, engad. vender, friaul. vendi, frz., prov., kat. vendre, sp., pg. vender. - Ablt.: it. vendita, frz. vente, pg. venda «Verkauf»; it. rivendugliolo «Höker», «Wiederverkäufer».

\subsubsection{Article */'ßend-e-/ du DÉRom}

*/'ßend-e-/ v.ditr. « céder (à qn) la propriété (de qch.) pour un certain prix »

*/'ßend-e-re/ > sard. bèndere/béndiri v.ditr. « céder (à qn) la propriété (de qch.) pour un certain prix, vendre » (DES ; PittauDizionario 1 ; AIS 825), dacoroum. vinde (dp. 1500/1510 [date du ms.], Psalt. Hur.2 175 ; Tiktin $_{3}$; EWRS ; DLR ; Cioranescu n 9274 ; MDA ; ALR SN 1018 ; 1926-1927 ; 2006 ; 2057), istroroum. vinde (MaiorescuIstria 156 ; Byhan,JIRS 6, 381 ; PuşcariuIstroromâne 3, 329 ; SârbuIstroromân 298 ; ALR SN 1018 ; 1926-1927 ; 2006 ; 2057), méglénoroum. vindiri (Candrea,GrS 7, 223 ; CapidanDicţionar s.v. vind ; Atanasov-

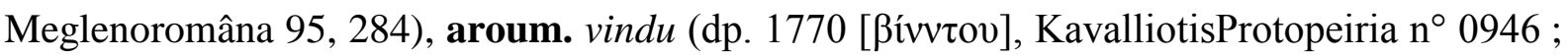
Pascu 1, 185 s.v. vindire ; DDA 2 ; BaraAroumain ; ALR SN 1926-1927 ; 2006 ; 2057) ${ }^{1}$, dalm. vander (BartoliDalmatisch 2, 103 ; ElmendorfVeglia; MihăescuRomanité 106), istriot. ‘véndi` (AIS 825), it. vendere (dp. 1207/1208, TLIOCorpus ; DELI2 ; AIS 825), frioul. vendi (PironaN 2 ; GDBTF ; AIS 825), lad. vëne (dp. 1763 [vanne], Kramer/Boketta in EWD ; AIS 825 ; ALD-I 846), romanch. vender (HWBRätoromanisch; AIS 825), fr. vendre (dp. 1160/1174, TLF ; Gdf ; TL ; FEW 14, 231b-232a; $\mathrm{AND}_{2}$; ALF 1358) ${ }^{2}$, frpr. ' vindre (dp. déb. $14^{\mathrm{e}}$ s. [vendre], DocLyonnais $188=$ HafnerGrundzüge 89 ; FEW 14, 232a ; HafnerGrundzüge 89, 126, 128, 131 [vendre] ; ALF 1358 ; ALLy 1247*), occit. vendre (dp. ca 1120, BrunelChartes 21; Raynouard ; Levy ; AppelChrestomathie ; Pansier 5 ; BrunelChartesSuppl 4 ; FEW 14, 231b-232a ; ALF 1358), gasc. 'béne’ (dp. av. 1256 [beno prét. 3], CartBigRC 121-122 ; FEW 14, 232a ; ALF 1358 ; ALG 1785 ; 1944-1947), cat. vendre (dp. $11^{\mathrm{e}}$ s. [uenuda part. p. f.], DCVB ; DECat 9, 99), esp. vender (dp. $2^{\mathrm{e}}$ m. $10^{\mathrm{e}}$ s., DCECH 5, 768 ; Kasten/Cody ; DME ; Kasten/Nitti) ${ }^{3}$, ast. vender (dp. 1240 [uender], DELIAMs ; DGLA), gal./port. vender (dp. 1220, TMILG ; DRAG2; DDGM ; DELP 3 ; Houaiss ; CunhaVocabulário 2 ).

Commentaire. - Tous les parlers romans sans exception présentent des cognats conduisant à reconstruire protorom. */'ßend-e-/ v.ditr. « céder (à qn) la propriété (de qch.) pour un certain prix, vendre ». 
Le corrélat du latin écrit, vendere v.ditr. «id. », est usuel durant toute l’Antiquité (dp. Plaute [* ca $254-\dagger 184]$, OLD).

Bibliographie. - MeyerLübkeGRS 1, § 70, 89, 94-97, 328, 332, 405 ; $\mathrm{REW}_{3}$ s.v. vēndĕre ; Ernout/Meillet $_{4}$ S.v. uēnum ; LausbergSprachwissenschaft 1, § 128, 169 ; 2, § 301 ; 3, § 787790 ; von Wartburg 1958 in FEW 14, 231b-234a, VĒNDĚRE ; Faré nº 9190 ; HallPhonology 42 ; SalaVocabularul 540.

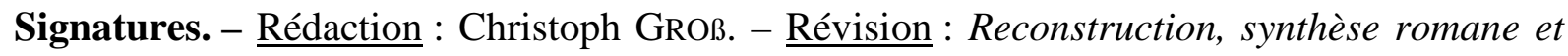
révision générale: Jean-Pierre Chambon. Romania du Sud-Est: Victor CelaC. Italoromania: Maria ILIESCU; Paul VIDESOTT. Galloromania: Jean-Paul CHAUVEAU. Ibéroromania : Ana Boullón ; Ana María CANo GonzÁLEz. Révision finale : Éva Buchi. Contributions ponctuelles: Petar AtAnasov; Cristina Florescu; Xosé Lluis García ArIAS ; Günter Holtus ; Fernando SÁNCHEz MIRET ; Uwe SCHMIDT.

Date de mise en ligne de cet article. - Première version : 03/01/2014. Version actuelle : 30/08/2014.

1. L'aroumain ne connaît presque plus l'infinitif verbal (cf. Saramandu,Tratat 460 ; Kramer,LRL 3, 429-430); la forme citationnelle est la première personne du singulier du présent. L’infinitif víndire (cf. l’entrée de Pascu citée ci-dessus) témoigne de l'appartenance du verbe à la flexion en */'-e-/, même s'il a connu par ailleurs un passage idioroman à celle en p

2. La première attestation fournie par le TLF est extraite de la Passion de Clermont, texte composé dans un idiome dont l'identification n’est pas assurée (peut-être occitan, $c f$. DePoerck,RLiR 27 ; DEAFBiblEl s.v. PassionA).

3. Les issues espagnoles, asturiennes, galiciennes et portugaises des verbes appartenant à la flexion en */'-e-/ du protoroman ont subi régulièrement une réaffectation à celle en */-'e-/ ou en */-'i-/ (cf. MeyerLübkeGLR 2, § 119, 126 ; WilliamsPortuguese § 148 ; LloydLatin 451455).

\subsubsection{Article tītio, -ōne du $\mathrm{REW}_{3}$}

8758. tītio, -ōne «Feuerbrand»

Rum. tăč̆une, it. tizzone, log. tittone, uengad. tizzun, friaul. stitsón, frz. tison, prov., kat. tizó, sp. tizón, pg. tição. - Ablt.: it. tizzo; nuor. bibyeḍ̣u, log. tintyeddu, campid. sittsyeddu «Ruß», «Getreidebrand» Wagner 149, 1; sp. tiznar, pg. tisnar «rußig werden», sp. tizna, pg. tisna «Ruß», sp. tizona «Schwert des Cid», vgl. 1273. - Zssg.: lucc. stizzare «auslöschen» Salvioni, Agl. 15, 199, viar. stizzorare «das Licht putzen», venez. stitsar, friaul. stisá «das 
Feuer anschüren», ait. stizzo, friaul. stitz «Feuerbrand»; vgl. 769. - + *EXTUTARE 3110: tessin., bergell. stüttsá Merlo, ID. 3, 298. - Diez 320 ; Baist, Zs. 5, 559. (It. stizza «Zorn», auch «Pips der Hühner» (als Sitz des Zornes) Prati, AGl. 18, 439, stizzire «reizen» Canello, AGl. 3, 404 gehören nicht hierher, das Verbum wird vielmehr eine Schallbildung sein.)

\subsubsection{Article */ti'tion-e/ du DÉRom}

*/ti'tion-e/ s.m. «morceau de bois incandescent; maladie des céréales d’origine cryptogamique qui les convertit en poussière noirâtre "

\section{Sens « tison "}

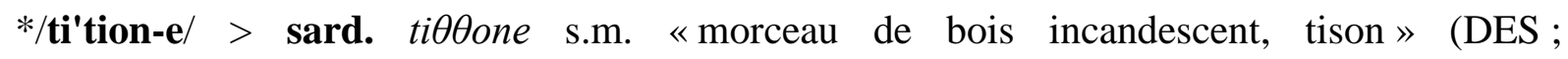
PittauDizionario 1 ; AIS 925), dacoroum. tăciune (dp. 1620, Tiktin 3 ; EWRS ; Cioranescu n ${ }^{\circ}$ 8443 ; DLR ; MDA ; SalaPhonétique 166, 225 ; ALR SN 1214 p 182, 250, 346, 520, 848) istroroum. tačuru (PuşcariuIstroromâne 3, 136 ; FrăţilăIstroromân 1, 293) ${ }^{2}$, méglénoroum. tăčuni (Candrea,GrS 7, 208 ; CapidanDicţionar s.v. tătšiuni ; AtanasovMeglenoromâna 196, 201, 283) ${ }^{3}$, aroum. tăciune (Pascu 1, 168 ; $\mathrm{DDA}_{2}$; BaraAroumain) ${ }^{4}$, it. tizzone (dp. 1288, GAVI ; Merlo,AUTosc 44, 85 ; DELI ; RohlfsGrammStor 1, § 289 ; TLIOCorpus ; AIS 925), frioul. stiçon (GDBTF s.v. stiç ; PironaN 2 [“Aggiunte e correzioni” s.v. stithon] ; AIS 925 p 328-329 ; ASLEF 399 n 1708 [«brûlure d'estomac »]) , lad. 'tizon’ (dp. 1879, Kramer/Fiacre in EWD s.v. tìza ; AIS 925 p 307, 310-311, 316, 322-323, 332), romanch. tizun (HWBRätoromanisch ; AIS 925 p 9-10, 13, 17, 19), fr. tison (dp. ca 1180 [tisun], TLF ; FEW 13/1, 356ab ; Gdf ; GdfC ; TL ; AND 1 s.v. tisun ${ }^{1}$; ALF 1721), frpr. 'tezon’ (dp. 1276 [tison], DevauxEssai 70 ; FEW 13/1, 356ab ; ALF 1721), occit. 'tizon' (dp. ca 1060 [tizun c.s. pl.], SFoiHA 1, 333 = Levy; Raynouard; AppelChrestomathie ; FEW 13/1, 356ab ; ALF 1721), gasc. 'tisoû' (Raynouard ; FEW 13/1, 356b ; Palay ; CorominesAran 720 ; ALF 1721), cat. tió (dp. ca 1271/1274, DCVB ; MollSuplement nº 3234 ; DECat 8, 495), esp. tizón (dp. 1235, DME ; Kasten/Cody; DCECH 5, 512 ; NTLE), ast. tizón (dp. 17 $7^{\mathrm{e}}$ s., DELlAMs ; DGLA), gal. tizón/port. tição (dp. ca 1260 [tiçon], DDGM ; DRAG 1 [sens secondaires] ; $\mathrm{DELP}_{3} ;$ Houaiss).

\section{Sens « charbon (maladie des céréales) »}

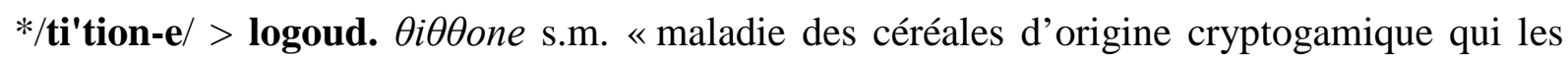
convertit en poussière noirâtre, charbon » (DES) ${ }^{6}$, dacoroum. tăciune (dp. ca 1650, DLR ; Tiktin $_{3}$; EWRS ; Cioranescu n 8443 ; MDA ; ALR SN 111), aroum. tăciune (Pascu 1, 168 ; $\mathrm{DDA}_{2}$ ), cal. tizzune (DTC) ${ }^{7}$, sic. tizzuni (VS [Capizzi, province de Messina]), frioul. stiçon (comm. pers. William Cisilin ; Valentino de Bean), esp. tizón (1801, CORDE), ast. tizón (DGLA ; DELIAMs). 
Commentaire. - À l'exception du dalmate, toutes les branches romanes présentent des cognats conduisant à reconstruire protorom. */ti'tion-e/ s.m. «morceau de bois incandescent, tison ; maladie des céréales d’origine cryptogamique qui les convertit en poussière noirâtre, charbon ${ }^{8}$.

Les issues romanes ont été subdivisées ci-dessus selon les deux sens qu'elles présentent : «tison » (I.) et «charbon (maladie des céréales) » (II.). Le premier sens, très majoritaire, couvre l'ensemble de l'espace occupé par le lexème, tandis que le second est restreint au roumain, au calabrais (pour le salentin, $c f$. n. 6), au sicilien, au sarde, au frioulan, à l'espagnol et à l'asturien. Le second sens peut également être reconstruit dans la protolangue, car il se retrouve dans les trois grands ensembles génétiques de la Romania : le sarde, le roumain et la Romania italo-occidentale ( $c f$. Jud,R 50, 606 ; FEW 13/1, 359a). En outre, le fait qu'il soit restreint à des aires latérales à l’échelle de la Romania (roum. esp. ast.) ou de l'Italie (calabr. sic. sard. frioul.), voire à des aires à la fois latérales et isolées (sic. sard.), révèle son ancienneté. Si l'analyse aréologique ne permet donc pas de déterminer la chronologie des sens, l'analyse sémantique amène à considérer le sens « charbon (maladie des céréales) » comme un développement métaphorique à partir du sens «tison »; en effet, le charbon donne aux plantes un aspect carbonisé9.

Le corrélat du latin écrit de I., titio, -onis s.m. «tison », est connu depuis Varron (* 116 - † 27, OLD ; “mot populaire d’après Lactance”, Ernout/Meillet4) ${ }^{10}$. Le latin écrit de l’Antiquité ne connaît pas, en revanche, de corrélat de II.

Bibliographie. - MeyerLübkeGLR 1, § 118-119, 135, 306-307, 350, 404-405, 450, 454, 509 ; $\mathrm{REW}_{3}$ s.v. tītio, -ōne; Ernout/Meillet4 s.v. tìtiō, -ōnis ; LausbergLinguistica 1, § 179-182, 231-235, 253, 273, 304, 405, 452-455; Müller 1966 in FEW 13/1, 356a-359b, TīTIO; HallPhonology 148 ; SalaVocabularul 543 ; MihăescuRomanité 255.

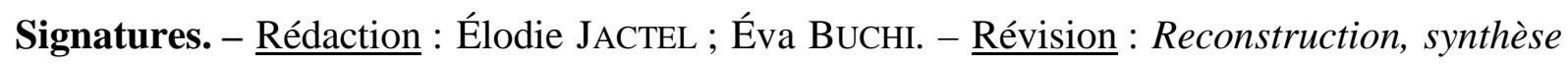
romane et révision générale: Jean-Pierre CHAmBon. Romania du Sud-Est : Victor CELAC ; Cristina FlORESCU. Italoromania : Giorgio CADORINI ; Rosario COLUCCIA ; Paul VIDESOTT. Galloromania: Jean-Paul CHAUveAu. Ibéroromania : Maria Reina BASTARDAS I RUFAT ; Myriam Benarroch; Ana Boullón; Ana María CANo GonzÁlez. Révision finale: Wolfgang SCHWEICKARD. - Contributions ponctuelles : Georges DARMS ; Xosé Lluis GARCíA ARIAS ; Günter Holtus ; Maria Iliescu ; Nikola Vuletić.

Date de mise en ligne de cet article. - Première version : 18/06/2012. Version actuelle : $31 / 08 / 2014$. 
1. La date de 1591 avancée par Tiktin 3 correspond à une attestation où tăciune représente un anthroponyme.

2. Avec changement de déclinaison idioroman.

3. Le genre féminin indiqué par CapidanDicţionar est erroné : son exemple atteste le masculin.

4. L’initiale d'istriot. 'steîso' (PellizzerRovigno ; AIS 925 p 368, 378, 397-398 ; ILA nº 581) oriente vers une analyse en tant qu'emprunt au frioulan.

5. Frioul. stiçon présente une prothèse de /s-/, qui constitue un phénomène très diffusé en frioulan (cf. Ascoli,AGI 1, 531-532).

6. Avec assimilation régressive (DES).

7. $C$. aussi le dérivé salent. tizzunara s.f. «id. »(VDS), qui présuppose l'existence ancienne du simple.

8. Dans certains idiomes de la Romania centrale (it. frioul. lad.), les issues de */ti'tion-e/ ont été ressenties comme des dérivés, ce qui a généré l’apparition de rétroformations de type 'tizzo’ (cf. Kramer/Fiacre in EWD s.v. tìza).

9. It. carbone s.m. «maladie de diverses plantes » (dp. 1759, Suani/Tressel/Hohnerlein in LEI 11, 1439, CARBO I $2 \mathrm{e}^{1} \alpha$ ) et fr. charbon (dp. 1701, TLF ; Poppe in FEW 2, 358b, CARBO III 3) représentent des évolutions sémantiques à partir de noms de maladies humaines (bubon et anthrax). On ne peut donc pas établir de parallèle sémantique direct entre ces dénominations de maladies de plantes et le sens II. de */ti'tion-e/, ce qui incite à écarter l’hypothèse d'un développement métaphorique spontané d'époque idioromane.

10. Le protoroman ne connaît pas de corrélat (ø $\mathrm{REW}_{3}$; ø FEW) de lat. torris « tison » (“mot rare et poétique”, Ernout/Meillet 4 s.v. torreō).

\subsubsection{Article crēscĕre du REW}

2317. crēscĕre « wachsen »

Rum. creşte, vegl. kraskro, it. crescere, log. kreskere, engad. krešer, friaul. krési, frz. croître, prov. creiser, kat. créixer, sp. crecer, pg. crescer. - Ablt.: bologn. karserit «Art Kuchen» Goidanich 35; neap. krišete, siz. krišenti, gen. krešente, viver. ksent, piem. kersent, velletr. krískuru «Hefe»; afrz. crestine, norm. kretin, kertin «das plötzliche Anschwellen einer Flusses».

\subsubsection{Article */'kresk-e-/ du DÉRom}

*/'kresk-e-/ v.intr./tr. "grandir progressivement jusqu’au terme du développement normal, croître ; rendre (qch.) plus grand, accroître »

\section{Verbe intransitif : « croître »}


*/'kresk-e-re/ > sard. krèskere v.intr. "grandir progressivement jusqu'au terme du développement normal, croître » (DES ; PittauDizionario 1), dacoroum. creşte (dp. 1500/1510 [date du ms.], Psalt. Hur.2 175 ; Tiktin3 ; EWRS ; Candrea-Densusianu nº 407 ; DA ; Cioranescu n 2562 ; MDA), istroroum. creşte (MaiorescuIstria 119 ; Byhan,JIRS 6, 254 ; PopoviciIstria 102 ; PuşcariuIstroromâne 3, 108, 184, 307 ; SârbuIstroromân 201 ; ScărlătoiuIstroromânii 299; ALIstro $n^{\circ}$ 1001), méglénoroum. creáştiri (CapidanDicţionar s.v. cres ; Candrea,GrS 3, 203-204 ; WildSprachatlas 480), aroum. créscu (dp. ca 1760 [că se crească], Kristophson,ZBalk 10/1 nº 0758 ; KavalliotisProtopeiria $n^{\circ} 0878$; DDA 2 ; Pascu 1, 70 ; BaraAroumain) ${ }^{1}$, dalm. crascro (BartoliDalmatico 316, 396, 430 ; ElmendorfVeglia), istriot. crìsi (DeanovićIstria 112 ; PellizzerRovigno ; ILA no 1001), it. crescere (dp. $2^{\mathrm{e}} \mathrm{m} .12^{\mathrm{e}}$ s., Ravani in TLIO ; DELI ${ }_{2}$ ), frioul. cressi (dp. 1365/1381 [cresin prés. 6], BenincàEsercizi 24 ; Rizzolatti in DESF ; GDBTF ; ASLEF $38 n^{\circ}$ 269, $430 n^{\circ}$ 1919-1920), lad. crësce (dp. 1763 [crusche], Kramer/Schlösser in EWD ; ALD-I 202), romanch. crescher (Decurtins in DRG 4, 236-240 ; HWBRätoromanisch), fr. croître (dp. ca 1100 [creistre], RolS2 142 = TLF ; Gdf ; FEW 2, 1323b ; TL ; AND 2 s.v. crestre ; DMF2012 ; ALF 362), frpr. creitre (dp. ca 1220/1230 [crestra], ProsalegStimm 55 ; HafnerGrundzüge 123 ; FEW 2, 1323b ; ALF 362), occit. creisser (dp. ca 1060 [creiss prés. 3], SFoiHA 1, 323 ; Raynouard; AppelChrestomathie 197 ; Levy; Pansier 3), gasc. creisser (dp. 1279 [creisseran fut. 6], DAG n 387 ; FEW 2, 1323b ; Palay; CorominesAran 418 ; ALF 362 ; ALG 2106), cat. créixer (dp. $1^{\text {ère }}$ m. $13^{\mathrm{e}}$ s., DECat 5, 1036-1038; MollSuplement $\mathrm{n}^{\circ} 1029$; DCVB), esp. crecer (dp. fin $12^{\mathrm{e}}$ /déb. $13^{\mathrm{e}}$ s. [creçe prés. 3], MenéndezPidalCid 2, 605 ; DCECH 2, 234235 ; Kasten/Cody ; DME) ${ }^{2}$, ast. crecer (dp. 1256 [crezca subj. prés. 3], DELIAMs ; DGLA), gal./port. crecer (dp. 1220/1240, TMILG ; DDGM ; Buschmann ; DRAG 1 ; DELP 3 [creçer] ; CunhaÍndice ; Houaiss ; CunhaVocabulário 2$)^{3}$.

\section{Verbe transitif : « accroître »}

*/'kresk-e-re/ > sard. krèskere v.tr. « amener à son plein développement, élever » (DES), dacoroum. creşte (dp. $16^{\mathrm{e}}$ s., Tiktin 3 ; Candrea-Densusianu $\mathrm{n}^{\circ} 407$; DA ; Cioranescu $\mathrm{n}^{\circ}$ 2562), méglénoroum. creáştiri (WildSprachatlas 480 [kreşte]), aroum. créscu ( $\mathrm{DDA}_{2}$; Pascu 1, 70), istriot. crìsi «rendre (qch.) plus grand, accroître » (PellizzerRovigno), it. crescere (dp. $1^{\text {ère }}$ m. $13^{\mathrm{e}}$ s. [ha cresciuto p. comp. 3], Ravani in TLIO) ${ }^{4}$, frioul. cressi (GDBTF), fr. croître (dp. $1^{\text {ère }}$ m. $12^{\mathrm{e}}$ s. [crestrai fut. 3], GormB 13 = TL ; Gdf ; GdfC ; FEW 2, 1323b ; $\mathrm{AND}_{2}$ s.v. crestre; DMF2012 ; TLF [aujourd'hui vieux et rare]) $)^{5}$, frpr. creitre (dp. 1286/1310 [creisit prét. 3], MargOingtD 106 ; GPSR 4, 598 ; FEW 2, 1323b), occit. creisser (dp. 1137/1152 [creis prés. 3], CercT 184 ; Raynouard; AppelChrestomathie ; FEW 2, 1323b), gasc. creisser (dp. 14 ${ }^{\mathrm{e}}$ s. [creysser], ArchHistGironde 11, 35 ; ForsBéarnOG 188, 
488 ; Palay), acat. créixer (1272 [crex prés. 3] - $2^{\mathrm{e}}$ t. $15^{\mathrm{e}}$ s., DCVB ; DECat 5, 1036-1038), aesp. $\operatorname{crecer}\left(13^{\mathrm{e}}\right.$ [creçer] - déb. $17^{\mathrm{e}}$ s., Kasten/Cody ; DCECH 2, 235), ast. crecer (DALIA), port. crecer (dp. $14^{\mathrm{e}}$ s., CunhaVocabulário 2 ; Houaiss) ${ }^{6}$.

Commentaire. - Tous les parlers romans sans exception présentent des cognats incitant à reconstruire protorom. */'kresk-e-/ v.intr. «grandir progressivement jusqu'au terme du développement normal, croître », tr. « rendre (qch.) plus grand, accroître » ${ }^{7}$.

Les issues romanes ont été subdivisées selon leur sémantisme et leur valence : verbe absolutif et intransitif (ci-dessus I.) et causatif et transitif (ci-dessus II.). En dépit d'une diffusion non complètement homogène et d'attestations généralement plus tardives du type II., nous y voyons un héritage commun : à l'exception du dalmate, du ladin et du romanche ${ }^{8}$, toutes les branches romanes connaissent, au moins au Moyen Âge, un emploi transitif du verbe, ce qui nous fait postuler que protorom. */'kresk-e-/ avait les caractéristiques d'un verbe labile (cf. CreisselsSyntaxe 2, 4 ; Letuchiy,Challenges 247).

Le corrélat de I. en latin écrit, crescere v.intr. " croître », est connu durant toute l’Antiquité (dp. Caton [*234 - † 149], TLL 4, 1176), tandis que le latin écrit de l’Antiquité ne connaît pas de corrélat du type $\mathrm{II}^{9}$. En revanche, le sens principal de crescere, «naître, venir au monde », usuel durant toute l’Antiquité (dp. Ennius [* 239 - † 169], TLL 4, 1176), est étranger aux cognats romans (cf. aussi DOLR 1, 56-57).

Bibliographie. - MeyerLübkeGLR 1, § 306-307, 313, 404-405, 438, 455, 468, 473, 532 ; 2, § 160 ; REW 3 s.v. crēscěre ; Ernout/Meillet 4 s.v. crēscō ; von Wartburg 1945 in FEW 2, 1323a1330a, CRĒSCĚRE ; LausbergLinguistica 1, § 168, 170, 337, 353-356 ; HallPhonology 751 ; SalaVocabularul 540 ; DOLR 1 (1991), 14 ; MihăescuRomanité 214.

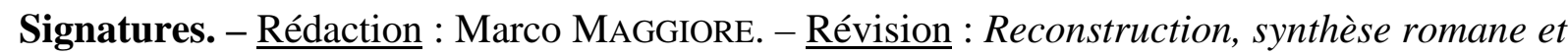
révision générale: Jean-Pierre CHAMBon. Romania du Sud-Est: Petar ATANAsov; Cristina FlORESCU. Italoromania: Giorgio CADORINI; Rosario COLUCCIA; Paul VIDESOTT. Galloromania: Jean-Paul ChAUVEAU. Ibéroromania : Maria Reina BASTARDAS I RUFAT; Myriam Benarroch ; Ana Boullón; Ana María CANo GonzÁlez. Révision finale : Éva BuCHI. - Contributions ponctuelles: Simone Augustin; Pascale BAUdinOT; Jérémie Delorme ; Xosé Lluis García Arias ; Xavier Gouvert ; Yan Greub ; Günter Holtus ; Stella MEdORI ; Jan ReINHARDT ; Mélynda SALCEDO ; Simone TrABER.

Date de mise en ligne de cet article. - Première version : 24/06/2011. Version actuelle : 04/09/2014. 
1. L'aroumain ne connaît presque plus l'infinitif verbal (cf. Saramandu,Tratat 460 ; Kramer,LRL 3, 429-430) ; la forme citationnelle est la première personne du singulier du présent.

2. L'espagnol, l'asturien et le galego-portugais présentent un déplacement d'accent régulier, dû à la perte de la classe flexionnelle en */'-e-/ (cf. MeyerLübkeGLR 2, § 126 ; LausbergLinguistica 2, § 788).

3. En portugais contemporain, cette graphie phonétique, courante jusqu'au $16^{\mathrm{e}}$ siècle et encore attestée jusqu'au $19^{\mathrm{e}}$ siècle (DDGM ; $\mathrm{DELP}_{3}$; Morais ${ }_{10}$ ), a été évincée par la variante $<$ crescer>, qui montre une influence savante. Contrairement à ce que suggère $\operatorname{DELP}_{3}$, cette dernière graphie n’apparaît pas seulement au $17^{\mathrm{e}}$ siècle, mais est attesté dès 1295/1312 (crescia, TMILG).

4. L’emploi transitif de crescere se trouve plus fréquemment au Moyen Âge. Pour ce qui concerne la langue contemporaine, il n'est courant que dans la locution crescere i figli (cf. AgenoVerbo 28-29), tandis qu'il est assez fréquent dans les dialectes méridionaux ( $c f$. RohlfsGrammStor 3, § 635).

5. Selon FEW 2, 1329a n. 2, le verbe transitif est ressenti comme une licence poétique depuis le début du $18^{\mathrm{e}}$ siècle.

6. L’emploi transitif du verbe est rare et, semble-t-il, limité au portugais du Brésil.

7. Bret. kreski (FEW 2, 1328b) semble avoir été emprunté à protorom. */'kresk-e-/.

8. Decurtins in DRG 4, 238 signale deux exemples d'emplois transitifs de romanch. crescher, dans l'acception «augmenter (la paye)». Il s'agit toutefois de cas isolés probablement idioromans.

9. La première attestation disponible de l'emploi transitif dans le code écrit se rencontre en 841/843 (MltWb).

Éva Buchi

ATILF (CNRS \& Université de Lorraine)

\section{Discussion}

\section{Réponse aux questions de Monsieur Martin :}

Monsieur l'Académicien, je vous remercie bien chaleureusement pour ces questions fort intéressantes ; j'essaierai d'y répondre de façon succincte. (1) Faire référence à la notion de type, comme vous le proposez, me semble très pertinent. Ce qui frappe, toutefois, en comparant les étymons trouvés par la méthode comparative avec ceux mis en avant par la méthode latinisante, c’est leur caractère plus varié (et donc moins abstrait), comme le montrent les exemples */'lumen/ */'lum-e/ */'lumin-e/ */'lumin-a/ */lu'min-a/ (Georgescu 2014 in DÉRom s.v. */'lumen/) face à lūmen $\left(\mathrm{REW}_{3}\right)$, */'molg-e-/ */'mong-e-/ 
(Delorme 2014 in DÉRom s.v. */'molg-e-/) face à mŭlgēre $\left(\mathrm{REW}_{3}\right)$ ou encore */re'tund-u/ */ro'tonnd-u/ */to'rond-u/ */'tond-u/ (Hegner 2011-2014 in DÉRom s.v. */re'tond-u/) face à rŏtŭndus $\sim$ rĕtŭndus $\left(\mathrm{REW}_{3}\right)$. (2) Pour ce qui est de votre seconde observation, il est en effet tentant de voir dans certains étymons de l'étymographie prédéromienne (comme dans *imprōmŭtŭare, $\mathrm{REW}_{3}$ ) le résultat d'une reconstruction comparative. Toutefois, quand on y regarde de plus près, on s’aperçoit qu'il n’en est rien: la série de cognats dacoroum. împrumuta/itsept. ' 'imprümüdar`/romanch. impermidar/fr. emprunter/frpr. 'inprontà`/occit. enprumtar/gasc. emprountà conduit à reconstruire protorom. */Im'prumut-a-/ (cf. Maggiore 2014 in DÉRom s.v.) et non pas **/Impro'mutu-a-/ : *imprōmŭtŭare représente du "fiddled with” classical Latin ( $f$. ci-dessus note 5) sur la base du verbe attesté promutuari « emprunter ». (3) Votre analyse des raisons profondes du rejet des pratiques du DÉRom par une partie des romanistes me semble très juste : pour beaucoup d'entre eux, c'est la notation en alphabet phonétique international des étymons qui pose problème. Ce choix de l'API s'explique d'une part, comme vous le remarquez à juste titre, par le caractère non biunivoque des conventions graphiques latines, d'autre part par notre volonté d'insister sur le caractère oral des étymons. Fort heureusement, la publication sous forme électronique du dictionnaire permet cependant, au moins partiellement, de réconcilier les deux approches : la consultation du dictionnaire par corrélats latins (decem, herba, pensum) et par entrées du $\mathrm{REW}_{3}$ (děcěm,

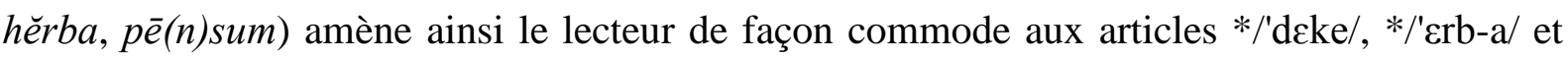
*/'pes-u/.

\section{Réponse à la question de Monsieur de Lamberterie :}

Monsieur l'Académicien, je vous remercie vivement pour cette question, qui touche au cœur de notre pratique étymologique - et concerne probablement la plus-value la plus importante du projet par rapport à ses prédécesseurs. Les étymons mis en avant par le DÉRom laissent en effet penser que le lexique protoroman présentait une assez grande variation interne. L'article */'cder-a/ s.f. « lierre » distingue ainsi les types I. */'eder-a/, II. */'cler-a/ et III. */'clen-a/, que le commentaire explique comme suit : « La large diffusion interromane des trois types et leur coprésence dans un seul domaine linguistique, l’occitan (“carrefour des langues romanes”), montrent que I., II. et III. sont des types déjà concurrents en protoroman et non des développements idioromans. Pour ce qui est de la chronologie relative des trois types, l'analyse formelle montre que I. est la base de II. (assimilation à distance partielle) et que II. est la base de III. (dissimilation à distance). Au plan aréologique, le roumain, l'espagnol, le portugais (aires latérales Est et Ouest) d'un côté, et le français et le francoprovençal (aire conservatrice Nord) de l'autre présentent le type I. Le type II. apparaissant en italoroman et dans ses périphéries septentrionales (dalmate, frioulan, ladin, romanche), on peut conclure 
qu'il s'agit ici d'une innovation venue d'Italie et étendue à la Narbonnaise et à partir de là en gascon, dans une aire plus ou moins centrale de la Romania. Le type III. apparaît seulement dans des domaines qui connaissent II. (italien, ladin, occitan), mais non dans tous, ce qui soutient l'idée d'une seconde innovation sur la base de II., plus tardive et donc moins largement diffusée » (Reinhardt 2010-2014 in DÉRom s.v. */'عder-a/). Comme le montre cet exemple, la méthode comparative est non seulement en mesure de reconstruire différents types, mais aussi leur chronologie relative.

\section{Réponse aux questions de Monsieur Pottier :}

Monsieur l'Académicien, je vous remercie bien chaleureusement pour vos questions. (1) La cartographie représente en effet un outil fort utile en étymologie, et le DÉRom y recourt très fréquemment. Il s’agit toutefois pour l'instant de cartes artisanales, dressées à la main, dont le but est exclusivement heuristique et non pas illustratif : elles ne présentent pas un caractère suffisamment abouti pour être publiées. Deux tentatives récentes montrent l’usage illustratif qui pourrait être fait de la cartographie en étymologie héréditaire (Maggiore et Buchi 2014, p. 316 ; Benarroch et Baiwir 2014, p. 142, 145-147, 150-152, 158-159), et suite à votre aimable suggestion, l'équipe du DÉRom s'efforcera de trouver une solution pour introduire sporadiquement des cartes dans le dictionnaire même. (2) Concernant votre seconde question, il est vrai que, dans la mesure où la reconstruction se fait sur la base d'unités lexicales orales, les cognats devraient idéalement être cités en transcription phonologique. Si nous y avons renoncé, c'est pour des raisons purement pratiques, et à regret (cf. Buchi et Schweickard 2011a, p. 307-308). 